\title{
Effect of remote ischemic preconditioning on myocardial injury after off-pump coronary artery bypass graft: unicentric randomized trial
}

\author{
Teresa Tejerina ${ }^{1}$, Monica Bouza ${ }^{2}$, Bunty Ramchandani ${ }^{3}$, Manuel Carnero $^{3}$, Victoria Acedo $^{3}$, \\ Fernando Reguillo ${ }^{3}$, Luis Maroto ${ }^{3}$
}

\author{
${ }^{I}$ Departament of Pharmacology, School of Medicne. U Complutense Madrid, Spain, ${ }^{2}$ Servicio de Cirugía Cardiaca, \\ Hospital Clínico San Carlos. Madrid. Departament of Pharmacology, School of Medicne. U Complutense Madrid, \\ Spain, ${ }^{3}$ Servicio de Cirugía Cardiaca, Hospital Clínico San Carlos. Madrid., Spain
}

Background / Study Objective: Remote ischemic preconditioning (RIP) on cardiac surgery has given variable results in different studies. We designed a clinical trial to evaluate the effect of RIP on myocardial injury after off-pump coronary artery bypass grafting (CABG). However, the effect of the cardiopulmonary bypass as well as the partial understanding of the mechanism of action can hide the potential cardioprotective effect. We also studied clinical effects and the signaling molecules involved: To compare levels of Troponin 12 hours after surgery between two groups (RIP CABG vs Control) and to compare clinical events and protein quantification involved between the two groups

Methods: Patients (67)* with indication to surgical myocardial revascularization off-pump were selected. Randomization $(1: 1)$

* RIP CABG (n: 31) Control (n: 36)

Age (years) 66.2 (1.34) 62.7 (1.73)

Male 25 (80.7\%) 33 (91.7\%)

Diabetes Mellitus 16 (51.6\%) 20 (55.6\%)

Hypertension 22 (71\%) 24 (66.7\%)

Smoking 4 (12.9\%) 5 (13.9\%)

Dyslipidemia 18 (58.1\%) 20 (55.6\%)

Logistic EuroSCORE 2.4 (1.2-3.6) 2.1 (1-3.2)

Results: see attached graphics .

Exclusion criteria were: urgency, concomitant procedures, previous myocardial infarction, haemodynamic inestability, liver dysfunction, renal failure and peripherical vasculopathy. The RIP was performed with 3 cycles of $5 \mathrm{~min}$ ischemia and $5 \mathrm{~min}$ reperfusion in an upper and lower limb using a blood pressure cuff inflated to $200 \mathrm{mmHg}$. We evaluated the troponin levels 6,12,24 hours after surgery and at discharge. The proteins quantification were obtained at basal and 6, 24 hours after surgery with Western Blot method. We evaluated postoperative clinical events: renal failure and atrial fibrillation.

Conclusion: The remote ischemic preconditioning after off-pump coronary artery bypass grafting in the upper and the lower limbs simultaneously hasn't demonstrated reduce the myocardial injury and neither to have repercussion in the new onset of atrial fibrillation or in acute renal failure. Cardioprotective proteins tend to be expressed more in patients receiving remote ischemic preconditioning with a peak at 6 hours after surgery. Limitations: Single center, not total recruitment, different anaesthetic protocol, few analysed samples. 